\title{
Arthroscopic Medial Patellofemoral Ligament
} Insertion Reconstruction and Plication for Medial Patellar Retinaculum Using Suture Anchor in First Acute Patellar Dislocation

\section{Tao Xu}

Second Clinical Medical College of China Three Gorges University: Three Gorges University Renhe Hospital

Liuhai Xu

Second Clinical Medical College of China Three Gorges University: Three Gorges University Renhe Hospital

Xinzhi Li

Second Clinical Medical College of China Three Gorges University: Three Gorges University Renhe Hospital

You Zhou ( $D$ zhouyou8010@163.com )

Second Clinical Medical College of China Three Gorges University: Three Gorges University Renhe Hospital

\section{Research Article}

Keywords: First acute patellar dislocation, Medial retinaculum, Medial patellofemoral ligament, Lateral release, Arthroscopy, Suture anchor

Posted Date: October 27th, 2021

DOI: https://doi.org/10.21203/rs.3.rs-1012304/v1

License: (c) (1) This work is licensed under a Creative Commons Attribution 4.0 International License. Read Full License 


\section{Abstract}

Background: The objective of this study was to evaluate the clinical results of arthroscopic medial patellofemoral ligament (MPFL) insertion reconstruction and plication for medial patellar retinaculum using suture anchor combined with lateral retinacular release in first acute patellar dislocation (APD) with MPFL insertion injury in adolescents.

Methods: A prospective study was performed between January 2016 and July 2019. The series included 61 cases of adolescent patients with first APD. There were 7 males and 54 females with an average age of 15.5 years ( 10 to 22 ). All cases were treated with arthroscopic suture anchor plication for medial patellar retinaculum combined with lateral retinacular release. Congruence angle (CA), lateral patellar angle (LPA), and patellar tilt angle (PTA) are measured by CT scan between last follow-up and preoperative. In addition, the patients were evaluated with the Lysholm and Kujala scores.

Results: The average follow-up time was 40.9 months (24-60 months). All 61 knees showed excellent or good results postoperatively. The Lysholm score increased significantly from $58.6 \pm 8.1$ to $91.9 \pm 5.0$ at the last follow-up postoperatively $(P<0.001)$. The Kujala scores increased significantly from $60.4 \pm 7.3$ to $88.9 \pm 4.8$ at the last follow-up postoperatively $(P<0.001)$. CA in $0^{\circ}$ extension position was improved significantly from $19.8 \pm 2.1^{\circ}$ preoperatively to $-6.7 \pm 1.7^{\circ}$ at the last follow-up $(P<0.001)$, LPA was increased from $-7.4 \pm 2.2^{\circ}$ to $5.7 \pm 1.8^{\circ}(P<0.001)$, and PTA was increased from $23.8 \pm 2.9^{\circ}$ to $12.3 \pm 2.3^{\circ}(P<0.001)$. The postoperative mean Lysholm and Kujala scores were 91.9 (81-100) and 88.9 (79-100), respectively.

Conclusions: When the first APD occurs associated with the MPFL avulsed from the patella, the presented technique could not only reattach MPFL at the patellar border but also strengthen the medial patellar retinaculum. This anatomical repair technique can significantly improve the stability of the patella and has the advantage of being less invasive by the full-arthroscopic approach.

\section{Introduction}

Acute patellar dislocation (APD) often occurs in children and adolescents, accounting for $9 \%-16 \%$ of traumatic joint injuries, and its management is controversial [1]. The medial patellofemoral ligament (MPFL) plays an important role in resisting lateral patellar displacement, especially in the knee flexion between $0-30^{\circ}$. Biomechanical studies have shown that MPFL contributes to about $50 \%$ of the restraint. The MPFL injury is one of the important causes of patellar instability and recurrent patellar dislocation [2]. Studies on nonoperative treatment report a high incidence of recurrent dislocations that have prompted some surgeons towards surgical treatment [3-5]. The MPFL repairment or reconstruction is the main surgical method in treating patellar dislocation when the bony anatomical abnormalities are excluded. Because acute primary patellar dislocation is more common in adolescents, and MPFL reconstruction is more traumatic and has the risk of epiphyseal injury. Yamamoto [6] describes the arthroscopic treatment of APD, which has the advantages of direct reefing of the medial retinaculum with minimally invasive surgery. However, these procedures become useless in the presence of a patellar 
insertion avulsion. Thus, a specific technique of MPFL reinsertion at the patellar border and meanwhile strengthening the medial patellar retinaculum seems to be more indicated [7-9]. These literatures only present technical descriptions and case reports with few follow-up studies. Therefore, the objective of this study is to evaluate the clinical results of MPFL insertion reconstruction and plication for medial patellar retinaculum by suture anchor combined with lateral retinacular release in APD with MPFL insertion injury in adolescents. Good clinical effects have been obtained, which are reported as follows.

\section{Methods}

From January 2016 and July 2019, 61 patients were treated by arthroscopic MPFL insertion reconstruction and plication for medial patellar retinaculum using suture anchor combined with lateral retinacular release. There were 7 males and 54 females with an average age of 15.5 years (10 to 22), 37 cases on the left knee, and 24 cases on the right knee. 43 cases were complicated with an avulsion fracture. Combined injuries included meniscus injury in 7 knees, contusion or exfoliation of lateral femoral condyle cartilage in 56 knees, loose body in 8 knees, medial collateral ligament injury in 2 knee, and anterior cruciate ligament injury in 1 knee. Cause of injury: all patients had a history of joint sprains or falls.

All patients were carefully evaluated with physical examination and with complete imaging examination. X-ray showed the general alignment of the patellofemoral joint, with or without fracture. CT scan was performed to observe lateral patella tilt and the avulsion fracture. MRI showed the injury site of MPFL, loose body, meniscus, cruciate ligament, etc.

Inclusion criteria: (1) avulsion fracture of the medial retinaculum near the medial edge of the patella as detected by CT and MRI, and then confirmed by arthroscopy; and (2) the first episode of APD, which is the important indication of this procedure (Fig. 1). Exclusion criteria: (1) recurrent patellar dislocation; (2) musculoskeletal disease or congenital bone deformity (such as severe trochlear dysplasia and knee valgus deformity); (3) medial retinaculum body and femoral side injury; (4) obvious increase of $Q$ angle; and (5) femoral anteversion or tibial rotation.

\section{Surgical Technique and Postoperative Rehabilitation}

The procedure was performed under epidural anesthesia and tourniquet control. Taking supine position, the body surface markers were drawn routinely before operation. First, conventional arthroscopy was performed through anteromedial and anterolateral approaches, and the hematoma and free body were removed at the same time. Complications, if any, such as meniscus and ligament injury should be treated at the same time. Then, the arthroscopic patellar trajectory is evaluated with repeated knee flexion and extension, and the injured MPFL insertion is freshened. Two absorbable suture anchors are placed at the trisection point of the medial patella. One end of the suture anchor is introduced into the articular cavity and passed through an anteromedial approach. The No. 1 polydioxanone suture (PDS suture) is passed through the needle, at the position of about $4 \mathrm{~cm}$ away from the medial patella, and then pull out the suture anchor from the anteromedial approach. Two or three sutures are then passed using this 
way.The lateral patellar retinaculum was released by the plasma cutter. Both ends of each suture are tightened through the subcutaneous skin until the patellar trajectory was recovered under arthroscopy postoperatively (Fig. 2, 4).

The knee joint was fixed in extension with brace for 4 weeks. Passive motion was $0-30^{\circ}$ in the first week, $0-60^{\circ}$ in the second week, $0-90^{\circ}$ in the third week, and reach normal level after 4 weeks. Physical exercise was resumed 3 months after the operation.

\section{Statistical analysis}

Statistical analysis was performed using the Statistical Package for the Social Sciences (SPSS) version 24.0. The measurement data were expressed as mean \pm standard deviation $(X \pm S)$. Preoperative and postoperative indices for this study were compared by paired t-test. $\mathrm{P}<0.05$ was defined as a significant difference.

\section{Results}

The average follow-up time of all patients was 40.9 months (24-60 months). At final follow-up, no patients suffered from patellar redislocation or subluxation. Apprehension signs remained in four patients. Three patient demonstrated restricted knee flexion of less than $90^{\circ}$ at two months postoperatively and regained a full range of motion after manipulation under physical therapy.

Significant knee function improvement, as assessed by the Lysholm and Kujala scores, was recorded at follow-up. The Lysholm scores were $91.9 \pm 5.0$ at the last follow-up, which were significantly higher than $58.6 \pm 8.1$ at preoperation $(t=26.876, P<0.001)$. The Kujala scores were $88.9 \pm 4.8$ at the last follow-up, which were significantly higher than $60.4 \pm 7.3$ at preoperation $(t=37.478, P<0.001)$ (Table 1$)$.

At final follow-up, the CT measurement results of CA, PTA, and LPA returned to the normal range (Fig. 3). CA decreased from $19.8 \pm 2.1^{\circ}$ preoperatively to $-6.7 \pm 1.7^{\circ}$ postoperatively $(t=77.633, P<0.001)$. LPA decreased from $-7.4 \pm 2.2^{\circ}$ preoperatively to $5.7 \pm 1.8^{\circ}$ postoperatively $(t=35.094, P<0.001)$, and PTA decreased from $23.8 \pm 2.9^{\circ}$ preoperatively to $12.3 \pm 2.3^{\circ}$ postoperatively $(t=26.179, P<0.001)$ (Table 2 ).

\section{Discussion}

The principal finding of the present study is that direct reinsertion of the MPFL with a suture anchor at the patellar border can obtain good imaging and functional outcome for the first APD in adolescents. Acute dislocations may cause avulsion of the medial patellofemoral ligamentous complex from the patella. Patients with APD have a high probability of MPFL injury, which mainly limits the lateral displacement of the patella. Yamamoto [6] first published his article on the arthroscopic repair of the MPFL for APD. Fukushima and Satterfield [7-9] presented cases of APD in a skeletally immature patient treated with arthroscopic medial patellofemoral ligamentous complex repair using suture anchors, this is amenable to direct primary repair to prevent recurrent instability and recreates normal anatomy and function. Ren et 
al. [10] reported that 12 patients with an avulsion fracture of medial retinaculum from the edge of the patella were repaired with suture anchor under ultrasonic positioning. The affected limb recovered quickly, the knee function was satisfactory, and there was no recurrence of patellar instability. Mariani et al. [11] treated 17 patients with first APD by repairing MPFL at the medial edge of the patella through a patellar tunnel. No redislocation occurred during follow-up. The Lysholm and Kujala scores after operation were significantly higher than those before operation. Fourteen out of 17 patients were able to return to sports at the same level as before. Toritsuka et al. [12] repaired MPFL patellar insertion point with suture anchor in 7 patients with primary patellar dislocation combined with MPFL patellar avulsion fracture. All patients recovered to the activity level before injury at the last follow-up, and there was no redislocation or subluxation. The above follow-up studies showed good postoperative function and no patellar instability. Relevant meta-analysis studies show that, the postoperative redislocation rate of surgical treatment is significantly better than that of conservative treatment. It is suggested that surgical treatment should be performed for the first patellar dislocation. Although reconstruction and repair are not discussed separately in the surgical treatment group, it seems that either type of surgery is superior to conservative treatment $[3,5]$. The incidence of redislocation caused by surgical treatment of primary APD (25\%) was significantly lower than that of conservative treatment (36.4\%) [13]. In a prospective randomized controlled study, the re-dislocation rate in the repair group $(22 \%)$ was significantly lower than that in the external fixation group (43\%) in children with primary traumatic APD [14].

However, this technique may not apply to all traumatic MPFL injuries with APD. One may achieve stable fixation of the MPFL by minimally invasive means provided several prerequisites were met such as proper patient selection, and exact localization of the lesion. Patients in the present study were confirmed as the primary APD which had a high healing rate and low tension when the MPFL was directly restored to its anatomical position. Accurate evaluation of the injury site of the medial retinaculum in APD played an important role in treatment decision-making. At present, the location of MPFL injury in APD is still controversial. Recent reports have indicated that patellar side avulsion has an appreciable incidence, contrary to previous studies which have characterized the injury as principally femoral side. The percentage of MPFL tears at the adductor tubercle was $73 \%$ of the cases studied [15]. Askenberger et al. [16] presented a study to describe MPFL injuries in the skeletally immature patient by MRI and to compare the results with the injury pattern found at arthroscopic surgery. The MRI scans showed an isolated MPFL injury at the patellar attachment site (60\%), a multifocal injury (35\%), an injury at the femoral site (4\%), and no injury (1\%). Arthroscopic surgery disclosed an isolated MPFL injury at the patellar site $(81 \%)$ and a multifocal injury (18\%). Skeletally immature children are more prone to sustaining an MPFL injury at the patellar attachment site. Another study reported by Seeley et al aimed to characterize the patterns of MPFL on MRI following primary APD in pediatric patients. MRI demonstrated MPFL injury in 87 patients (78.4\%). MPFL injury was present at an isolated patellar insertion (31\%), an isolated femoral insertion (14\%), and more than one location was present (33\%) [17]. Kepler et al. [2] reported that $61 \%$ of MPFL injuries occurred at the patellar attachment and $12 \%$ at the femoral attachment. Most studies showed that MPFL injury after patellar dislocation mainly occurs at the patellar side, which is consistent with our clinical data. 
Clarifying the injury location has important clinical guiding significance for the selection of treatment methods and surgical approaches. Evaluating the location of avulsion fracture at the medial edge of the patella is of guiding significance for the selection of anchor insertion point and puncture point of the medial retinaculum. Anatomical studies showed that MPFL was mainly located in the middle and upper part of the patella, and MPTL / MPML was mainly attached to the lower part of the patella [18]. Mochizuki et al. [19] reported that $84 \%$ of the patellar avulsion fractures were located in the inferomedial patellar border, which consisted of MPTL/MPML attachments but not the MPFL attachments that mainly limits the outward movement of the patella. This strange phenomenon may be caused by many factors, such as injury energy, buckling, and torsion direction. Among APDs, non-contact knee sprain in flexion and valgus is the leading mechanism. When the knee flexion is greater than $45^{\circ}$, excessive rotation, valgus, and inclination of the patella will cause excessive tension of MPTL and MPML at the medial lower edge of the patella. It is consistent with the mechanism that most of the patients with primary APD included in this study have a history of non-collision, flexion, and torsion injury [20]. However, only repairing the secondary limiting structures of MPTL and MPML located at the medial lower edge of the patella may not be enough to limit patellar Exodus, and MPFL is the main binding force to limit patellar exodus. Therefore, in this study, two anchors were placed at the trisection of the medial edge of the patella, and the medial retinaculum was sutured evenly. The follow-up results showed that there was no recurrent patellar instability. Biomechanical studies have shown that the repair of MPFL with suture anchor suture can almost produce similar contact stress and joint kinematics compared with the reconstruction of MPFL with tendon transplantation [21]. Some studies have shown that the incidence of re-dislocation after MPFL repair is equivalent to or better than that of allograft reconstruction [22]. Reconstruction has higher requirements for the surgeon, requires additional grafts, and may damage the epiphysis of children [23].

After the medial retinaculum was repaired and pre-knotted and tightened, we observed the trajectory of a patellofemoral joint under arthroscopy, and then decided whether to release the lateral retinaculum. The purpose was to avoid the high pressure between the patella and medial femoral condyle and the significant increase of medial instability caused by excessive release and increase the risk of iatrogenic medial dislocation. Or insufficient release caused excessive suture tension of medial anchor and instability of the patellofemoral joint, resulting in failure of healing of medial retinaculum reinforced suture [24]. The advantages of this operation were anatomical repair of MPFL insertion and plication for medial patellar retinaculum and simultaneous treatment of other structural injuries in the joint. The potential advantages of arthroscopic surgery for patellar dislocation are less trauma, less operative complications, and more intuitive release of lateral retinaculum to accurately adjust patellar trajectory under monitoring. Our follow-up results were satisfactory, and there was no recurrence and re-dislocation. This study also has some limitations. Firstly, due to the strict selection criteria, the number of patients included is limited. Secondly, there is a lack of an effective control group. Despite these limitations, this study hopes that surgeons will pay attention to the absence of MPFL avulsion fracture of the patella and the possibility of arthroscopic-assisted suture anchoring in the treatment of this injury. 


\section{Conclusion}

To sum up, in the case of the first APD, the tightening repair of the medial retinaculum complex can prevent the symptoms of recurrent patellar dislocation and patellar instability. Arthroscope-assisted minimally invasive surgery for the first APD, especially in adolescents with high incidence, can avoid epiphyseal injury, less trauma, significantly improve patellar stability and early recovery of knee joint function. To achieve good clinical results, comprehensive physical examination, imaging diagnosis, and arthroscopic confirmation must be performed before an operation to determine the exact location of the tear of the medial retinaculum, repair the dissecting stop and restore the tension balance of the medial and medial patella. Finally, our follow-up score and postoperative imaging were satisfactory.

\section{Abbreviations}

MPFL: arthroscopic medial patellofemoral ligament; APD: acute patellar dislocation; CA: Congruence angle; LPA: lateral patellar angle; PTA: patellar tilt angle; PDS: polydioxanone suture

\section{Declarations}

\section{Ethics approval and consent to participate}

This study was approved by the Institutional Review Board (IRB) of Affiliated Renhe Hospital of China Three Gorges University. The volunteer involved in the study consent to participate in the study. And the written consent has been obtained from the volunteer.

\section{Consent for publication}

All individual person's data consent to publish.

\section{Availability of data and materials}

Please contact author for data requests.

\section{Competing interests}

The authors declare that they have no competing interests.

\section{Funding}

None.

\section{Authors' contributions}

Tao Xu and You Zhou conceived and designed the experiments. Tao Xu wrote a draft of the manuscript. Liuhai Xu contributed to the data and produced the figures. You Zhou performed the surgical procedure. 
You Zhou and Xinzhi Li analyzed and interpreted the results of the experiments and revised the manuscript. All authors read and approved the final manuscript.

\section{Acknowledgements}

None.

\section{References}

1. Sanders T L, Pareek A, Hewett T E, Stuart M J, Dahm D L, Krych A J. Incidence of First-Time Lateral Patellar Dislocation: A 21-Year Population-Based Study. Sports Health. 2018,10(2):146-151.

2. Kepler C K, Bogner E A, Hammoud S, Malcolmson G, Potter H G, Green D W. Zone of injury of the medial patellofemoral ligament after acute patellar dislocation in children and adolescents. Am J Sports Med. 2011,39(7):1444-1449.

3. Migliorini F, Driessen A, Quack V, Gatz M, Tingart M, Eschweiler J. Surgical versus conservative treatment for first patellofemoral dislocations: a meta-analysis of clinical trials. Eur J Orthop Surg Traumatol. 2020,30(5):771-780.

4. Pagliazzi G, Napoli F, Previtali D, Filardo G, Zaffagnini S, Candrian C. A Meta-analysis of Surgical Versus Nonsurgical Treatment of Primary Patella Dislocation. Arthroscopy. 2019,35(8):2469-2481.

5. Yang F, Guo W, Wang Q, Zhu Z, Guan C, Zhao S, Yuan B. Surgical versus nonsurgical treatment of primary acute patellar dislocation: A systematic review and meta-analysis. Medicine (Baltimore). 2019,98(29):e16338.

6. Yamamoto R K. Arthroscopic repair of the medial retinaculum and capsule in acute patellar dislocations. Arthroscopy. 1986,2(2):125-131.

7. Fukushima K, Horaguchi T, Okano T, Yoshimatsu T, Saito A, Ryu J. Patellar dislocation: arthroscopic patellar stabilization with anchor sutures. Arthroscopy. 2004,20(7):761-764.

8. Satterfield W H, Johnson D L. Arthroscopic patellar "bankart" repair after acute dislocation. Arthroscopy. 2005,21(5):627.

9. Dodson C C, Shindle M K, Dines J S, Altchek D W. Arthroscopic suture anchor repair for lateral patellar instability. Knee Surg Sports Traumatol Arthrosc. 2010,18(2):143-146.

10. Ren M, Zhen P, Fang Y J, Ren X L, Dang C P, Hou F Y, Li S S. Study on therapeutic effects of arthroscopic repair on medial patellar retinaculum acute injury. Zhongguo Gu Shang. 2017,30(1):2932.

11. Mariani P P, Liguori L, Cerullo G, lannella G, Floris L. Arthroscopic patellar reinsertion of the MPFL in acute patellar dislocations. Knee Surg Sports Traumatol Arthrosc. 2011,19(4):628-633.

12. Toritsuka Y, Horibe S, Hiro-Oka A, Mitsuoka T, Nakamura N. Medial marginal fracture of the patella following patellar dislocation. Knee. 2007,14(6):429-433.

13. Longo U G, Ciuffreda M, Locher J, Berton A, Salvatore G, Denaro V. Treatment of Primary Acute Patellar Dislocation: Systematic Review and Quantitative Synthesis of the Literature. Clin J Sport 
Med. 2017,27(6):511-523.

14. Askenberger M, Bengtsson M E, Ekstrom W, Arendt E A, Hellsten A, Mikkelsen C, Janarv P M. Operative Repair of Medial Patellofemoral Ligament Injury Versus Knee Brace in Children With an Acute First-Time Traumatic Patellar Dislocation: A Randomized Controlled Trial. Am J Sports Med. 2018,46(10):2328-2340.

15. Putney S A, Smith C S, Neal K M. The location of medial patellofemoral ligament injury in adolescents and children. J Pediatr Orthop. 2012,32(3):241-244.

16. Askenberger M, Arendt E A, Ekstrom W, Voss U, Finnbogason T, Janarv P M. Medial Patellofemoral Ligament Injuries in Children With First-Time Lateral Patellar Dislocations: A Magnetic Resonance Imaging and Arthroscopic Study. Am J Sports Med. 2016,44(1):152-158.

17. Seeley M, Bowman K F, Walsh C, Sabb B J, Vanderhave K L. Magnetic resonance imaging of acute patellar dislocation in children: patterns of injury and risk factors for recurrence. J Pediatr Orthop. 2012,32(2):145-155.

18. Kruckeberg B M, Chahla J, Moatshe G, Cinque M E, Muckenhirn K J, Godin J A, Ridley T J, Brady A W, Arendt E A, Laprade R F. Quantitative and Qualitative Analysis of the Medial Patellar Ligaments: An Anatomic and Radiographic Study. Am J Sports Med. 2018,46(1):153-162.

19. Mochizuki T, Tanifuji O, Watanabe S, Katsumi R, Tomiyama Y, Sato T, Endo N. The majority of patellar avulsion fractures in first-time acute patellar dislocations included the inferomedial patellar border that was different from the medial patellofemoral ligament attachment. Knee Surg Sports Traumatol Arthrosc. 2020,28(12):3942-3948.

20. Philippot R, Boyer B, Testa R, Farizon F, Moyen B. The role of the medial ligamentous structures on patellar tracking during knee flexion. Knee Surg Sports Traumatol Arthrosc. 2012,20(2):331-336.

21. Mehl J, Otto A, Comer B, Kia C, Liska F, Obopilwe E, Beitzel K, Imhoff A B, Fulkerson J P, Imhoff F B. Repair of the medial patellofemoral ligament with suture tape augmentation leads to similar primary contact pressures and joint kinematics like reconstruction with a tendon graft: a biomechanical comparison. Knee Surg Sports Traumatol Arthrosc. 2020,28(2):478-488.

22. Bryant J, Pandya N. Medial patellofemoral ligament repair restores stability in pediatric patients when compared to reconstruction. Knee. 2018,25(4):602-608.

23. Shah J N, Howard J S, Flanigan D C, Brophy R H, Carey J L, Lattermann C. A systematic review of complications and failures associated with medial patellofemoral ligament reconstruction for recurrent patellar dislocation. Am J Sports Med. 2012,40(8):1916-1923.

24. Clifton R, $\mathrm{Ng} \mathrm{C} \mathrm{Y,} \mathrm{Nutton} \mathrm{R} \mathrm{W.} \mathrm{What} \mathrm{is} \mathrm{the} \mathrm{role} \mathrm{of} \mathrm{lateral} \mathrm{retinacular} \mathrm{release?} \mathrm{J} \mathrm{Bone} \mathrm{Joint} \mathrm{Surg} \mathrm{Br.}$ 2010,92(1):1-6.

25. Zhao J, Huangfu X, He Y, Liu W. Recurrent patellar dislocation in adolescents: medial retinaculum plication versus vastus medialis plasty. Am J Sports Med. 2012,40(1):123-132.

\section{Tables}


Table 1 Results of knee function during follow up.

\begin{tabular}{|lllll|}
\hline Variable & Preoperative & Last follow-up & $t$ Value & $P$ Value \\
\hline Lysholm score & $58.6 \pm 8.1$ & $91.9 \pm 5.0$ & 26.876 & $\bowtie 0.001$ \\
\hline Kujala & $60.4 \pm 7.3$ & $88.9 \pm 4.8$ & 37.478 & $\bowtie 0.001$ \\
\hline
\end{tabular}

Table 2 Patellofemoral morphology on CT measurements.

\begin{tabular}{|c|c|c|c|c|}
\hline Variable & Preoperative & Last follow-up & t Value & P Value \\
\hline Congruence angle $\triangle C A \rrbracket$, deg & $19.8 \pm 2.1^{\circ}$ & $-6.7 \pm 1.7^{\circ}$ & 77.633 & $\bigotimes 0.001$ \\
\hline Lateral patellar angleखLPA囚, deg & $-7.4 \pm 2.2^{\circ}$ & $5.7 \pm 1.8^{\circ}$ & 35.094 & $\otimes 0.001$ \\
\hline Patellar tilt angle $\triangle \mathrm{PTA} \rrbracket$, deg & $23.8 \pm 2.9^{\circ}$ & $12.3 \pm 2.3^{\circ}$ & 26.179 & $\bowtie 0.001$ \\
\hline
\end{tabular}

\section{Figures}
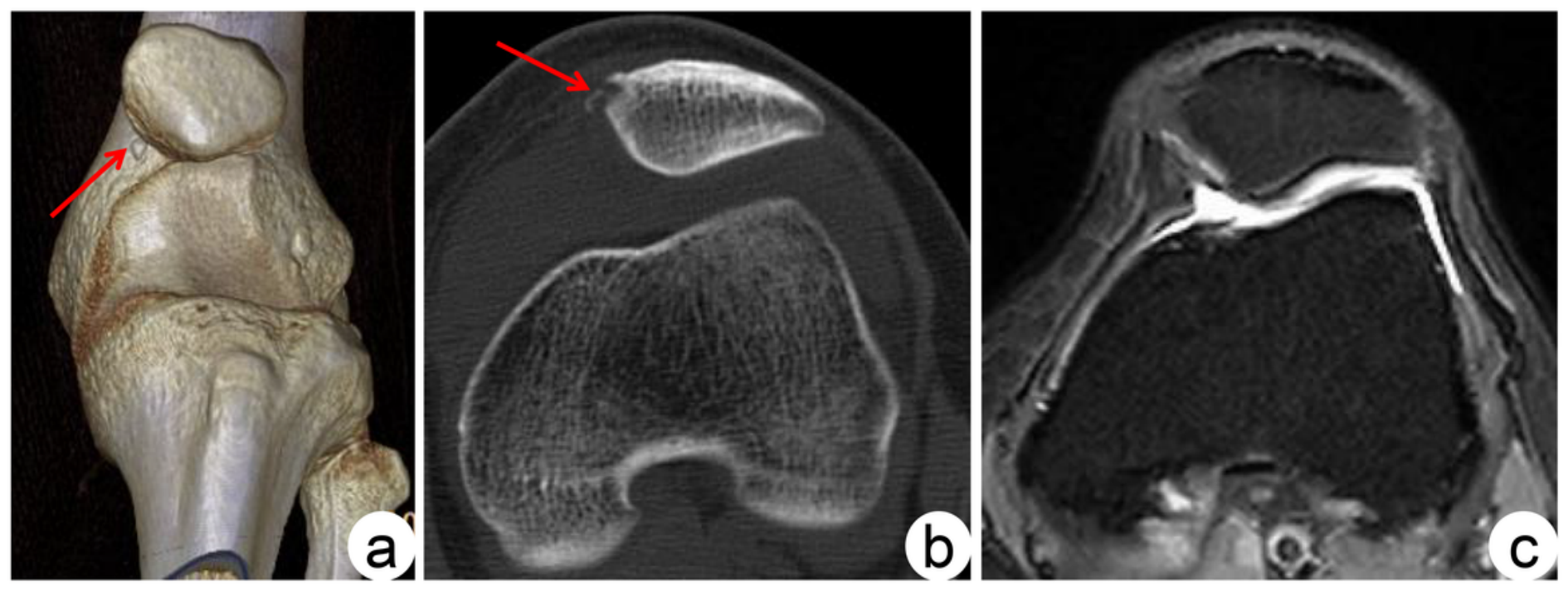

\section{Figure 1}

Preoperative imaging findings ab. Preoperative knee computer tomography (CT) showed lateral dislocation of patella with an avulsion fracture of the medial edge of patella. c. Preoperative knee magnetic resonance imaging (MRI) showed that ligament injury of medial patellar retinaculum with a patellar avulsion fracture. 


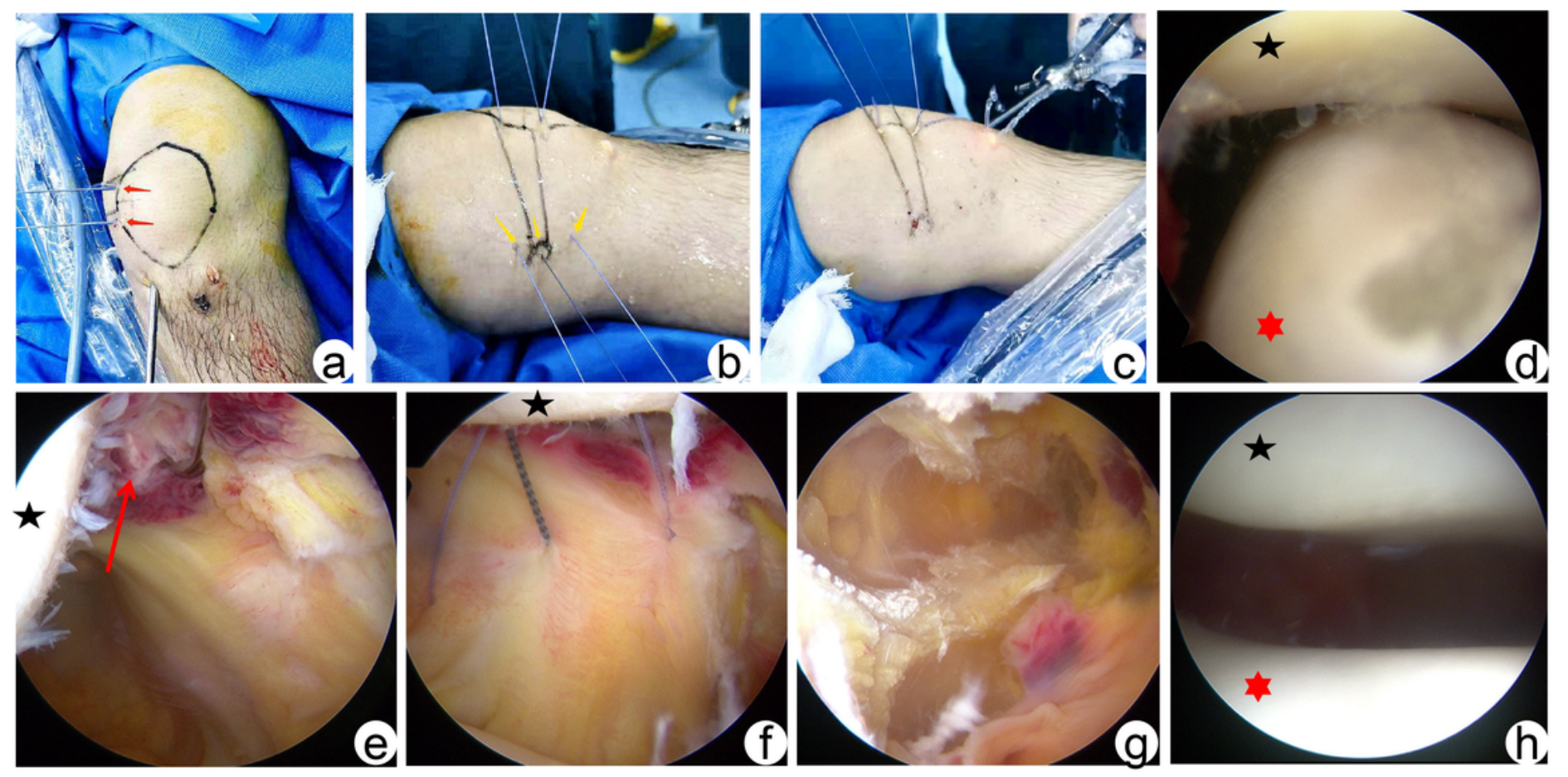

Figure 2

Intraoperative gross and microscopic views a. Draw the mark on the body surface, and the anchors were located at the trisection point of the patella (red arrow). b. The anchor suture passed through the medial retinaculum complex (yellow arrow). c. The suture passed subcutaneously and was pre-knotted at the medial edge of the patella. $d$. Lateral subluxation of the patella (black pentagram: patella; black hexagonal: lateral condyle of the femur). e. Medial retinaculum tear combined with patellar avulsion fracture (red arrow). f. The medial retinaculum complex was repaired with suture anchors. g. Release of the lateral retinaculum. $h$. The patellofemoral joint was well involuted. 

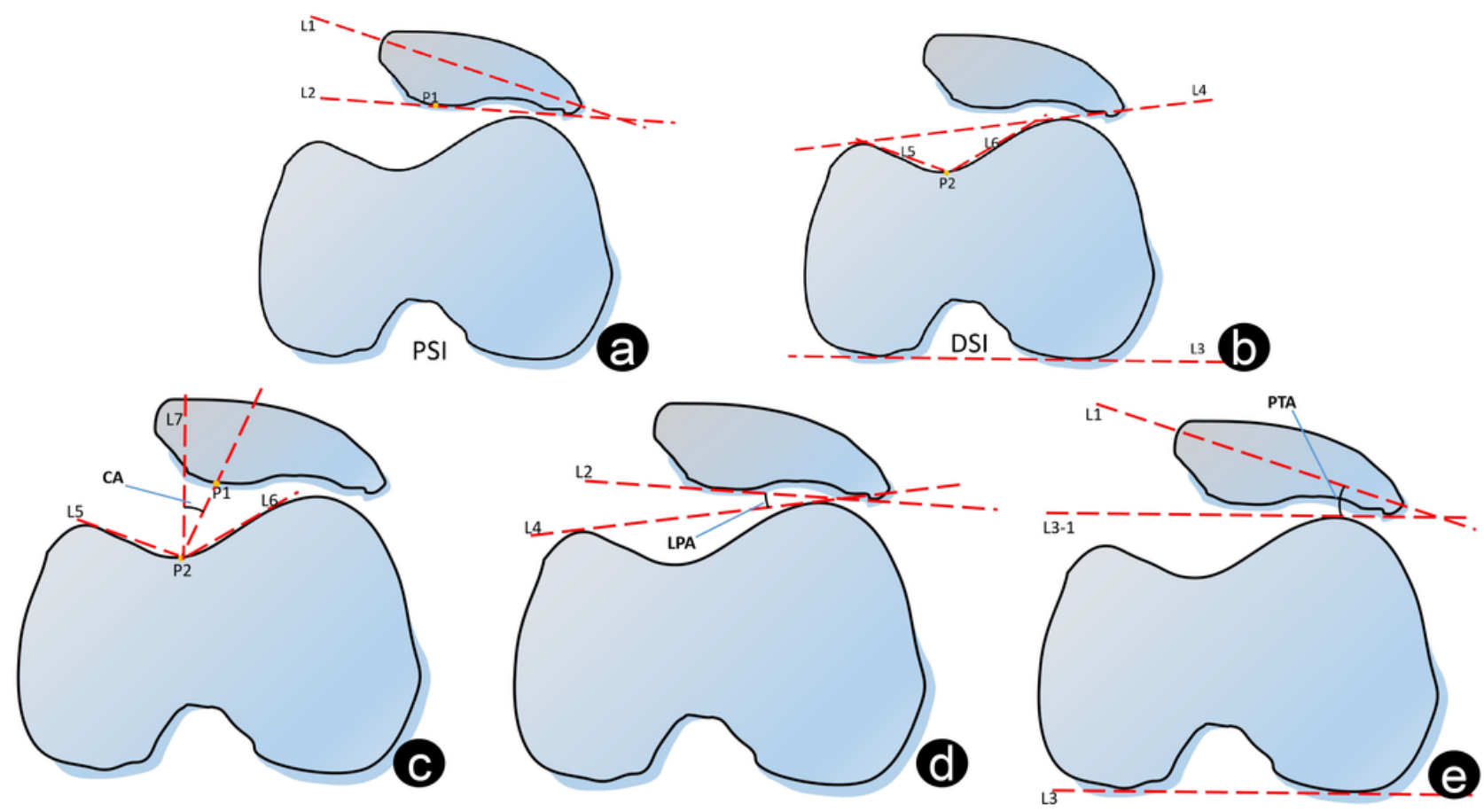

\section{Figure 3}

Schematic diagram of the operation a. Cross-sectional model: the suture anchor was placed into the medial edge of the patella (blue arrow), and the suture passed through the medial articular capsule and retinaculum complex. b. Lateral view pattern: two suture anchors were evenly placed on the medial edge of the patella (anchor, green arrow; medial retinaculum complex, red pentagram)
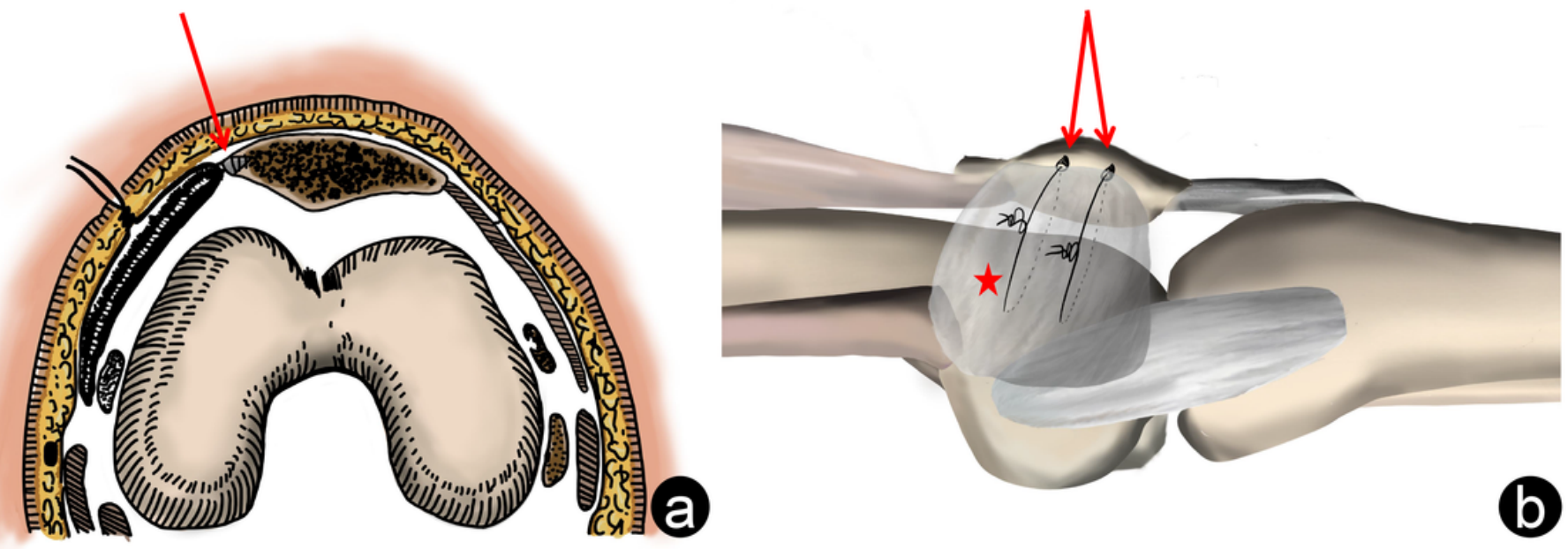

Figure 4 
a. Proximal standard image (PSI) of the patella in CT examination, which cut through the widest and thickest level of the patella. ( $L 1$, the transverse axis; $L 2$, lateral facet; $P 1$, the posterior apex of the patella). b. Distal standard image (DSI) of the patella in CT examination, which cut through the most prominent and distal level of the medial femoral condyle. (P2, the apex of the trochlea groove; $L 3$, the posterior tangential line of the femoral condyles; L4, the anterior tangential line of the femoral condyles; L5 and L6, the sulcus angle). c. The angle between L7 and P1P2 is the congruence angle (CA). L7 bisects the sulcus angle. $d$. The angle between L2 and L4 is lateral patellar angle (LPA). e.The angle between L1 and L3-1 is the patellar tilt angle (PTA). L3-1, a parallel line to L3[25]. 